\title{
Light Extraction via Leaky Modes in Organic Light Emitting Devices
}

\author{
Nils A. Reinke, Claudia Ackermann and Wolfgang Brütting ${ }^{1}$ \\ Experimental Physics IV, University of Augsburg, 86135 Augsburg, Germany
}

\begin{abstract}
Display and illumination technology require light sources with angular independent emission behaviour. Conversely, a strongly angular dependent spectral emission can be desirable for other applications in information technology or spectroscopy. In order to elucidate the potential of organic light-emitting devices (OLEDs) for the latter fields, we performed experimental and numerical studies of the angular dependent emission characteristics of cavity like OLEDs. The light generated in the organic multilayer structure and guided in leaky modes was coupled out by a prism. Here, a semitransparent gold anode, acting as a hole injection layer, was used to enhance the coupling of leaky modes guided inside the OLED to external modes (Kretschmann configuration). The observed light emission was strongly angle dependent, with the spectral emission peak of the device shifting from a wavelength of $680 \mathrm{~nm}$ to $500 \mathrm{~nm}$ as the angle is varied between $20^{\circ}$ and $70^{\circ}$ with respect to the normal of the substrate plane. Also, the emitted light shows a high degree of polarisation. The observed behaviour can be predicted quantitatively by simulations, which are based on the transfer matrix formalism.
\end{abstract}

\footnotetext{
${ }^{1}$ Corresponding author, e-mail: wolfgang.bruetting@physik.uni-augsburg.de
} 


\section{Introduction}

In standard organic light-emitting device (OLED) structures (see Fig. 1a) a large fraction of the generated light is trapped inside the organic layers or the substrate by total internal reflection, or is coupled to surface plasmons associated with the presence of metallic electrodes. Ultimately, this fraction is lost by emission at the device edges or reabsorption. A rough estimate shows that only about $20 \%$ of the generated photons can leave the device in forward direction [1-5]. There are several approaches reported in the literature, to raise the performance of OLED structures. Microlenses and scattering microspheres at the backside of the glass substrate can suppress the propagation of light in substrate modes [6,7]. Also the implementation of an aerogel layer sandwiched between the organic multilayer stack and the substrate can avoid the coupling to substrate modes [8]. Furthermore, the implementation of a periodic corrugation can be utilized to scatter out wave-guided light $[9,10]$ or extract energy from surface plasmons [11].

The problem of light extraction from OLEDs is related to the inverse situation of coupling electromagnetic radiation from an external source into guided modes, also termed leaky modes, inside an organic film or to surface plasmons at the interface between thin metal films and organic layers. A frequently used technique to achieve this is the grating coupler method $[12,13]$. Therein a grating is used to circumvent the refractive index mismatch, which prohibits the coupling of external to guided modes. An alternative way to excite surface plasmons or guided modes in organic thin films is the prism coupler method [14], which is often combined with a semitransparent metallic layer, sandwiched between the substrate and the active layer. The additional metal film facilitates the tunnelling of radiation from an external source into guided leaky modes in the organic layer and can therefore enhance the coupling performance. The essential idea in the context of OLEDs is that light has to take the same pathway to escape from the multilayer structure that is taken by the light coupled from an external source into related thin film structures. Thus, in this work, we have employed a prism coupler to extract energy from wave-guided leaky modes in OLEDs. Light, which is generated inside the multilayer structure and wave-guided as leaky modes or anodic surface plasmons, can leave the device by tunnelling through a semitransparent anode.

Moreover, in OLEDs, as opposed to the standard coupling experiments on neat organic films, one has to take into account that the semitransparent anode is forming a microcavity resonator together with the counterelectrode, which usually is a fully reflective cathode. Cavity OLEDs are among the most effective structures to enhance light output and enable bright and purecolour emission. As reported previously the light emission from microcavities is spectrally 
narrowed and displays a strong angular dependence of the peak wavelength [15-17]. It has been observed that the emission peak of cavity OLEDs can be shifted by as much as $100 \mathrm{~nm}$ in the optical range [18-21]. Typically, the spectrum shows a blue shift, with increasing angle of emission. By tuning the thicknesses of the organic layers this blue shift can be efficiently reduced [22,23]. In this work, we present an OLED architecture, which is colour tuneable from a wavelength of $500 \mathrm{~nm}$ to $680 \mathrm{~nm}$ by changing the viewing angle. Our devices exhibit a strongly s-polarized light emission (i.e. perpendicular to the plane of incidence), which is ten times more intense than light emission with p-polarization (parallel to the plane of incidence).

\section{Experimental}

In this work we compare two types of samples: As a reference device (A), we have used a standard two-layer OLED structure consisting of $80 \mathrm{~nm}$ of the organic hole transporter N,N'bis-(3-methylphenyl)-N,N'-bis-(phenyl)-benzidine (TPD) in combination with 80nm of the electron transport material tris-(8-hydroxyl-chinolinato)-aluminium $\left(\mathrm{Alq}_{3}\right)$, which also served as the emitter. This organic hetero-structure was sandwiched between a PEDOT:PSS (Baytron P, HC Starck GmbH, Germany) coated ITO anode on a glass substrate and a $\mathrm{Ca} / \mathrm{Al}$ cathode (see Fig. 1a). The so-called leaky-mode device (B) consisted of a borosilicate glass (BK 7) substrate on which a thin, semi-transparent Au layer was evaporated as the anode. Otherwise the structure was identical to (A) (see Fig. 1b).

Both substrates were cleaned by subsequent treatments for 15 minutes each in an ultrasonic bath of detergent, acetone and isopropanol. In the case of device (A), a $30 \mathrm{~nm}$ thick layer of the conducting polymer PEDOT:PSS was deposited by spin-coating as a hole-injection and buffer layer on top of a $125 \mathrm{~nm}$ thick ITO layer. For device (B), a $30 \mathrm{~nm}$ gold layer was deposited by thermal evaporation to form a semi-transparent anode. In order to raise the work-function of the gold film, the substrate was exposed to oxygen plasma. Subsequently the samples were coated in a vacuum of $10^{-7}$ mbar by evaporating an $80 \mathrm{~nm}$ thick hole-transportlayer of TPD, $80 \mathrm{~nm}$ of the electron-transport/emissive material $\mathrm{Alq}_{3}$, and $40 \mathrm{~nm}$ of calcium and $60 \mathrm{~nm}$ aluminium. Finally the samples were encapsulated by capping the multilayerstructures with glass slides and solvent free epoxy to allow for a characterization in ambient air.

The encapsulated devices were mounted on a rotary table so that the axis of rotation was coincident with the active area of the devices (Fig. 2). For measurements of the electroluminescence spectra the samples were driven at a constant current using a Keithley 220 current source. After passing through a polarizing filter to discriminate between s- and p- 
polarized light, the light was collimated into a fiber and spectrally analyzed using a diode array spectrometer (Ctf-60, CCDUV16 - 1024, Optische Messtechnik GmbH, Ulm, Germany). By turning the rotary table, we were able to take angular dependent emission spectra over a wide range of emission angles. This was accomplished by combining freespace detection of light emission (Fig. 2a) with light outcoupling through a prism (Fig. 2b). Whereas in the first case total internal reflection limits the range of observable emission angles to less than $41.5^{\circ}$, the latter technique allows to cover also large emission angles up to $70^{\circ}$. In this case the device substrate is attached to the cathetus of a right angle prism using an index matching gel and light emission is observed through the hypotenuse of the prism. When light is leaving the prism via the hypotenuse side, refraction of light at the glass/air interface has to be taken into account to obtain the emission angle $\varphi$ at the OLED. Alternatively a hemispherical lens with the light emitting device positioned in the centre of the lens can be used, which allows for a direct investigation of the radiative distribution inside the glass substrate [18]. However this configuration has a lower angular resolution as the emission area is extended. Finally, the light output integrated over all solid angles was measured with and without the prism in an integrating sphere equipped with a calibrated radiometric detector.

\section{Experimental Results}

Fig. 3 shows a colour map of the angle dependent emission of the ITO reference device (A) measured without an attached prism. The s- and p-polarized fractions of the emitted light are virtually identical. Their spectral shape is angle independent and the light intensity has a Lambertian angular distribution. In this geometry the external emission angles (ranging from $0^{\circ}$ to $90^{\circ}$ towards the normal of the substrate and measured at the substrate/air interface) correspond to internal emission angles at the anode/substrate interface between $0^{\circ}$ and $41.5^{\circ}$. As described above, a more extended range of internal emission angles from $15^{\circ}$ to $70^{\circ}$ can be investigated, if a prism is attached to the OLED. In Fig. 4 the corresponding colour maps of the light emission are shown. The s-polarized emission is apparently more intense than the ppolarized fraction and shows its highest values at an internal emission angle of about $40^{\circ}$ and a wavelength of $520 \mathrm{~nm}$. This is associated with the presence of a guided leaky mode, which radiates into the substrate as it propagates inside the organic layers. At the observed emission maximum the guided leaky mode fulfils the transversal resonance condition at a wavelength of about $520 \mathrm{~nm}$ which corresponds to the free-space emission peak of $\mathrm{Alq}_{3}$. As we will show below, this effect becomes more pronounced as the reflectivity of the adjacent electrodes is increased. For smaller emission angles the resonance condition is fulfilled at larger 
wavelengths, which is indicated by the red shift in the colour map in Fig. 4 with decreasing viewing angles. In comparison, the p-polarized light emission is much less pronounced and the spectrum is more or less angular independent. This indicates that radiation from guided leaky modes is not involved here and rather a direct emission of the p-polarized fraction occurs.

Fig. 5 shows the emission properties of the leaky-mode device (B) implementing gold instead of ITO as the anode material, measured without the prism. For both polarisation directions the vertical emission displays two emission peaks at $570 \mathrm{~nm}$ and $750 \mathrm{~nm}$. The red emission peak of the s-polarized spectrum is shifting from $750 \mathrm{~nm}$ to $630 \mathrm{~nm}$ and grows in intensity with increasing viewing angle to reach a broad maximum at an emission angle $\omega$ of about $65^{\circ}$ (corresponding to an internal angle of about $31^{\circ}$ ). By contrast, the green emission peak at 570 $\mathrm{nm}$ is slightly moving to longer wavelength with increasing angle and finally merges with the previous one in the broad maximum at $630 \mathrm{~nm}$. The p-polarized spectrum offers a similar picture, although the emission intensity is strongly reduced. As compared to the corresponding ITO device (Fig. 3), having basically identical emission spectra for both s- and p-polarisation with only one angular independent peak, the appearance of a second mode at longer wavelength together with a pronounced polarisation and angle dependence of the emission characteristics are a clear signature of microcavity effects in the leaky-mode device. Fig. 6 shows the emission properties of the same device (B) with an attached right angle prism. The emission is dominated by one peak with pronounced angle dependence and a high degree of polarisation. In the s-polarized spectrum the wavelength of the emission peak shifts from $680 \mathrm{~nm}$ to $500 \mathrm{~nm}$ by changing the emission angle $\varphi$ from $25^{\circ}$ to $65^{\circ}$. The overall maximum can be found at a wavelength of $550 \mathrm{~nm}$ and an emission angle of $53^{\circ}$. As compared to the reference device (A) in Fig. 4, the s-polarized emission of the leaky-mode device (B) is spectrally narrowed and has a stronger angular dependence. This can be attributed to a redistribution of oscillator strength inside the microcavity as a consequence of an increased anode reflectivity. Contrarily, the emission characteristics of the p-polarized light emission is only weakly structured, which can be explained by missing pathways of leakymode emission in the investigated range of angles. So, similar to device (A) the p-polarized emission of the leaky-mode device (B) passes direct. A direct comparison of s- and ppolarized light emission at a constant wavelength of $550 \mathrm{~nm}$ is presented in Fig. 7. Obviously, the s-polarized fraction of light emission is ten times more intense than the p-polarized fraction. By contrast, the reference device (A) shows a relatively week degree of polarization of only $20 \%$ at the same wavelength (see Fig. 4). 
From the comparison of all the emission characteristics discussed so far, it is evident that for both the ITO and the microcavity OLED the overall emission maximum is located at angles close to or even larger than $42^{\circ}$ and is thus cut off by total internal reflection in a device on a planar substrate. Therefore the attached prism allows gaining information on the internal emission properties of an OLED. Furthermore, we also expect an increase of the overall light output for both device types if a prism is used. In order to estimate the relative amount of light emitted in forward direction, trapped in substrate modes and coupled out of leaky modes we measured both the reference device (A) and the microcavity device (B) using an integrating sphere to collect the light emission over all solid angles. At first, we measured the light output originating from both forward and edge emission of the devices $\left(\mathrm{I}^{(0)}\right)$. Afterwards, we blackened the edges of the device substrates to suppress the latter fraction $\left(\mathrm{I}^{(1)}\right)$. Finally, we attached the device to the prism and measured again the integral light output $\left(\mathrm{I}^{(2)}\right)$. The obtained relative intensities are given in Table 1.

Assuming that all light from guided modes can be coupled out by using the prism [*], one can now extract the fraction of light emitted in forward direction $\mathrm{I}^{(1)} / \mathrm{I}^{(2)}$ and the one trapped in substrate modes 1 - $\mathrm{I}^{(1)} / \mathrm{I}^{(2)}$. From the latter, a fraction $\left(\mathrm{I}^{(0)}-\mathrm{I}^{(1)}\right) /\left(\mathrm{I}^{(2)}-\mathrm{I}^{(1)}\right)$ can leave the substrate via the edges, whereas the remaining part is reabsorbed by the multilayer stack. For the reference device (A) we find that $43 \%$ of the generated light intensity can leave the device in forward direction and $57 \%$ is guided in substrate modes. From these guided modes, about $56 \%$ can leave the device via the edges and $44 \%$ are reabsorbed. For the leaky-mode device (B), only $29 \%$ of the generated photons are emitted in forward direction and as much as $71 \%$ are trapped in substrate modes. This directly reflects the lower transmission of gold as compared to an ITO anode. Correspondingly, $41 \%$ of the trapped photons leave the device at the edges and $59 \%$ are reabsorbed.

It is evident from these numbers that the implementation of an optical element, in this case a prism, can enhance the overall light output from OLEDs considerably. However, as the comparison of both devices also shows, there is still room for further improvement in the case of the microcavity device. Mainly, due to the non-ideal optical properties of the gold anode this device still has three to four times lower efficiency even when a prism is used. However, we expect that replacing gold by another high-work function metal with superior reflectivity over the whole spectral range, e.g. silver with suitable surface treatment, should yield better light extraction. 


\section{Optical Modelling}

There are various techniques to calculate the emission characteristics of OLED structures, which can be divided into three general schemes: (1) The radiant molecules are treated as oscillating dipole antennas, which are embedded into a multilayer system. This classical approach allows to calculate the far field radiation of these dipoles [24,25]. (2) In the quantum mechanical description, the electromagnetic field of an OLED is given by the sum of eigenmodes, where the transition probability into these modes is determined by "Fermi's golden rule" [26]. (3) Another technique calculates the reflectance of OLEDs with and without an additional optical gain layer [27]. This layer represents the emissive zone of the OLED and the spectral gain resembles the photoluminescence spectrum of the emissive material. In particular, the latter model takes interference effects into account, which arise from refractive index discontinuities in such a multilayer stack. These interference effects are handled straightforward by the transfer matrix formalism, which is a frequently used tool to calculate the angle dependent transmission and reflectance of multilayer structures for both sand p-polarisation [28].

In order to model the emission spectra of our devices, we performed numerical simulations, which are also based on the transfer matrix formalism. The transfer matrix of our OLED structures is given by

$$
\left(\begin{array}{ll}
M_{11} & M_{12} \\
M_{21} & M_{22}
\end{array}\right)=D_{0}^{-1}\left[\prod_{l=1}^{N} D_{l} P_{l} D_{l}^{-1}\right] D_{S} .
$$

Therein $D_{0}$ and $D_{S}$ are the transmission matrices for the substrate and the cover, respectively, and $\mathrm{D}_{l}$ and $\mathrm{P}_{l}$ are the transmission and the propagation matrices for the $l$-th layer. These matrices include the thicknesses and the complex refractive indices of the different materials inside the multilayer structure. The complex refractive indices were obtained by spectral ellipsometry for the organic materials or extracted from literature data in the case of metals $[29,30]$. Then, the reflectance $R$ is given by

$$
R(\lambda, \varphi)=\left|\frac{M_{21}}{M_{11}}\right|^{2}
$$

A metal cathode of $100 \mathrm{~nm}$ thickness leads to a virtually opaque device, i.e. there is basically no transmission in the spectral range of interest here. Insofar, we obtain the absorbance $A$ of a given multilayer structure with zero transmission by using the conservation of energy

$$
A(\lambda, \varphi)=1-R(\lambda, \varphi)
$$

At maxima of $A$ for given sets of parameters $(\lambda, \varphi)$, light is coupled most effectively into guided leaky modes. In these modes, the light intensity is mainly concentrated in the organic 
layers and decaying exponentially into the metallic electrodes. Our model presumes that the same pathway taken by the radiation from an external light source to couple into leaky modes is used by photons generated inside the organic multilayer structure to leave the device. Thus, the spectral and angular dependent outcoupling efficiency of light from leaky modes is identical to the absorbance $A(\lambda, \varphi)$. The emission spectrum $I_{e m}(\lambda, \varphi)$ of an OLED is then given by the product of the spectral distribution of the generated photons $I_{0}(\lambda)$, i.e. the emission spectrum of the material, times the probability of the photons to leave the device as leaky modes:

$$
I_{e m}(\lambda, \varphi)=I_{0}(\lambda) \times A(\lambda, \varphi)
$$

Here, $I_{e m}$ is the angular and spectrally modulated emission of $\mathrm{Alq}_{3}$ embedded into a microcavity resonator. Since the excitation densities in our devices are relatively small, the internal electromagnetic fields do not influence the transition dipoles of the emissive material. We therefore take as the internally generated emission spectrum of $\mathrm{Alq}_{3}$ the electroluminescence spectrum emitted in forward direction of the ITO based reference OLED (A) with the same multilayer structure, where cavity effects are playing a minor role only. The recorded EL spectrum of the reference OLED (A) in forward direction is shown in Fig. 8.

Fig. 9 then displays the results of our calculations for the leaky-mode device (B). The simulation predicts a shift of the spectral maximum from $500 \mathrm{~nm}$ at an emission angle of $70^{\circ}$ to $680 \mathrm{~nm}$ at $20^{\circ}$, for the s-polarized case. In contrast, there is only a weak emission for the ppolarized fraction. The simulation also predicts a slight increase in intensity of p-polarized light at grazing angles. This can be traced back to extraction of radiation from surface plasmons travelling along the gold/TPD interface.

From the comparison with the experimental data shown in Fig. 6 one can clearly see that the transfer matrix formalism incorporating the spectral function of the emissive layer well describes the emission characteristics of our devices. Discrepancies at high and low angles can originate from reflection losses, when light passes the hypotenuse of the prism with an angle close to the angle of total reflection.

\section{Conclusions}

Our investigations show that in planar OLEDs a large fraction of the spectral emission is lost by total internal reflection and coupled to modes travelling inside the substrate. Therefore, we were using a prism to record almost the full angle dependent emission spectrum of our devices. This configuration enables us to study the light emission, which is usually going into wave-guided modes and finally reabsorbed or emitted at the device edges. We found, that the 
emission spectrum of the demonstrated cavity OLED displays a strong angular dependence and offers a high degree of polarization. Also we presented a technique to simulate the emission characteristics of multilayer OLED structures. It accounts for interference effects arising from multiple beam reflections at interfaces with different refractive indices inside the multilayer stack. Although the position and the extension of the emission zone are not addressed explicitly, we found a reasonable agreement with our experimental results.

Thus, the presented combination of a microcavity with an optical element offers various possibilities to optimize multilayer OLEDs with respect to light outcoupling efficiency and spectral emission properties as well as to achieve highly polarized light emission which can be favourable for certain applications in information technology. Furthermore, OLEDs have the potential to be used as integrated light sources in leaky-mode or surface plasmon spectroscopy. 


\section{References}

[1.] N. C. Greenham R. H. Friend and D. D. C. Bradley, ADV MAT 6, 491, 1994

[2.] J. S. Kim et al., J APPL PHYS 88, 1073, 2000

[3.] K. Meerholz and D. C. Müller, ADV FUNCT MATER 11, 251, 2001

[4.] N. K. Patel, S. Cina and J. H. Burroughes, IEEE JOURNAL ON SELECTED TOPICS IN QUANTUM ELECTRONICS 8, 346, 2002

[5.] G. Gu et al., OPT LETT 22, 396, 1997

[6.] C. F. Madigan, M. H. Lu and J. C. Sturm, APPL PHYS LETT 76, 1650, 2000

[7.] T. Yamasaki, K. Sumioka and T. Tsutsui, APPL PHYS LETT 76, 1243, 2000

[8.] T. Tsutsui et al., ADV MAT 13, 1149, 2001

[9.] J. M. Lupton et al., APPL PHYS LETT 77, 3340, 2000

[10.] N. Reinke et al., J LUMIN 110, 413, 2004

[11.] P. A. Hobson et al., ADV MAT 14, 1393, 2002

[12.] A. Perschke and T. Fuhrmann., ADV MAT 14, 841, 2002

[13.] H. Raether, "Surface Plasmons", Springer-Verlag Berlin Heidelberg, 1988

[14.] M. Osterfeld, H. Franke and C. Feger, APPL PHYS LETT 62, 2310, 1993

[15.] H. F. Wittmann et al., ADV MAT 7, 541, 1995

[16.] N. Takada et al., APPL PHYS LETT 63, 2032, 1993

[17.] V. Cimrova and D. Neher, J APPL PHYS 79, 3299, 1996

[18.] V. Bulovic et al., PHYS REV B 58, 3730, 1998

[19.] T. Nakayama, Y. Itoh, and A. Kakuta, APPL PHYS LETT 63, 594, 1993

[20.] A. Dodabalapur et al., APPL PHYS LETT 64, 2486, 1994

[21.] T. Tsutsui et al., APPL PHYS LETT 65, 1868, 1994

[22.] A. B. Djurisic and A. D. Rakic, OPT COMM 236, 303, 2004

[23.] C. H. Cheung et al., OPT COMM 248, 287, 2005

[24.] O. H. Crawford, J CHEM PHYS 89, 6017, 1988

[25.] K. A. Neyts, J. OPT. SOC. AM. A 15, 962, 1998

[26.] K. B. Kahen, APPL PHYS LETT 78, 1649, 2001

[27.] B. Ruhstaller et al., IEEE JOURNAL OF SELECTED TOPICS IN QUANTUM ELECTRONICS 9, 723, 2003

[28.] P. Yeh, “Optical Waves in Layered Media”, Wiley Interscience, 2005

[29.] L. Ward, "The Optical Constants of Bulk Materials and Films", $2^{\text {nd }}$ Ed., Institute of Physics Publishing, 1994 
[30.] E. D. Palik, "Handbook of Optical Constants of Solids", Academic Press INC. (LONDON) LTD., 1985

[*] We note that a hemispherical lense instead of a right-angle prism should give even higher overall light output. However, the extended active device area leads to a diminished angular resolution in this case. 


\section{Tables}

\begin{tabular}{|c|c|c|c|}
\hline & $\begin{array}{c}\mathrm{I}^{(0)} \\
\text { as fabricated }\end{array}$ & $\begin{array}{c}\mathrm{I}^{(1)} \\
\text { blackened edges }\end{array}$ & $\begin{array}{c}\mathrm{I}^{(2)} \\
\text { with prism }\end{array}$ \\
\hline Reference device (A) & 1 & 0.57 & 1.34 \\
\hline Leaky - mode device (B) & 0.26 & 0.15 & 0.52 \\
\hline
\end{tabular}

Table 1: Angle integrated light output of the reference device (A) and the leaky-mode device (B) measured in different configurations. All data are given as ratios relative to the light output of the reference device (A) without blackened edges and prism. 


\section{Figures}

(a)

\begin{tabular}{|c|c|}
\hline 60 nm & $\mathrm{Al}$ \\
\hline $40 \mathrm{~nm}$ & $\mathrm{Ca}$ \\
\hline $80 \mathrm{~nm}$ & $\mathrm{Alq}_{3}$ \\
\hline 80 nm & TPD \\
\hline $30 \mathrm{~nm}$ & PEDOT:PSS \\
\hline $125 \mathrm{~nm}$ & ITO \\
\hline
\end{tabular}

(b)

\begin{tabular}{|c|c|}
\hline $60 \mathrm{~nm}$ & $\mathrm{Al}$ \\
\hline $40 \mathrm{~nm}$ & $\mathrm{Ca}$ \\
\hline $80 \mathrm{~nm}$ & $\mathrm{Alq}_{3}$ \\
\hline $80 \mathrm{~nm}$ & TPD \\
\hline $30 \mathrm{~nm}$ & $\mathrm{Au}$ \\
\hline
\end{tabular}

Fig. 1: Schematic multilayer structure of the investigated OLEDs on glass substrates (BK 7): (a) reference device (A) with a transparent anode and (b) leaky-mode device (B) with semitransparent gold electrode. 
a)

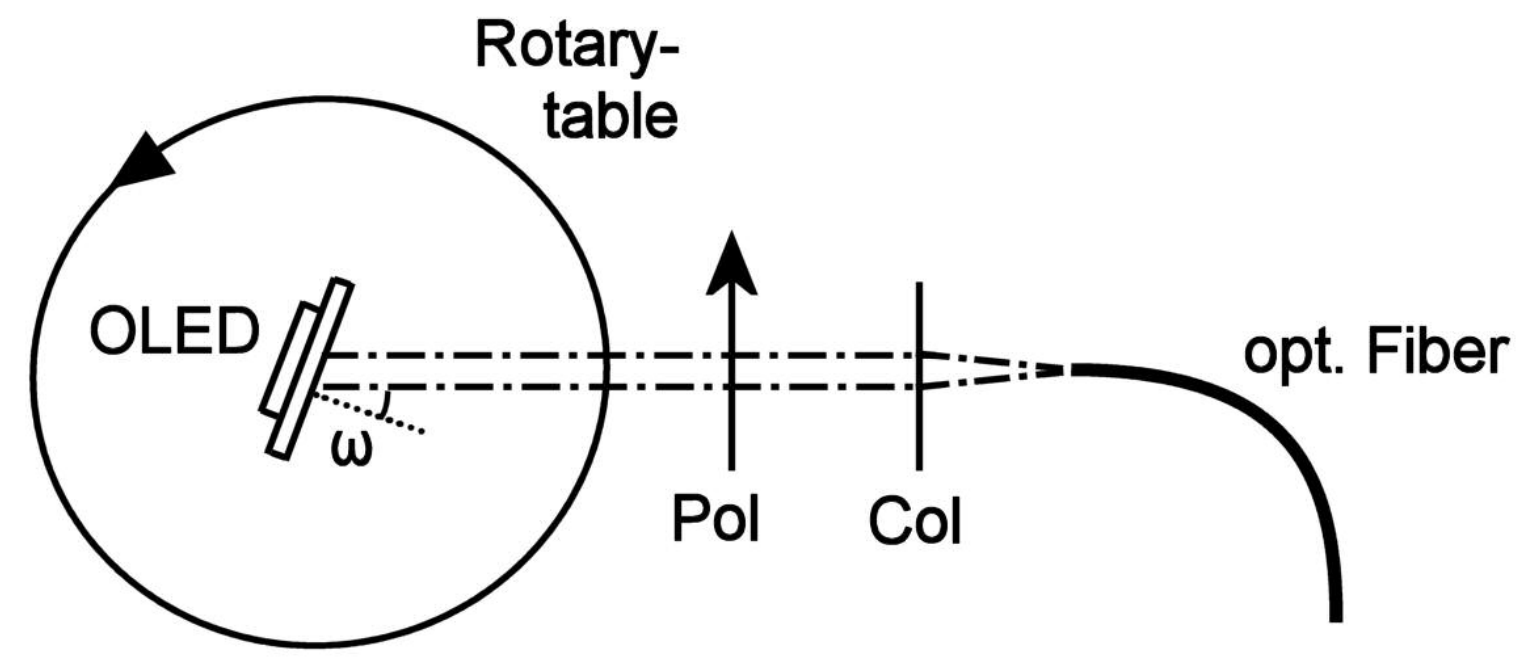

b)

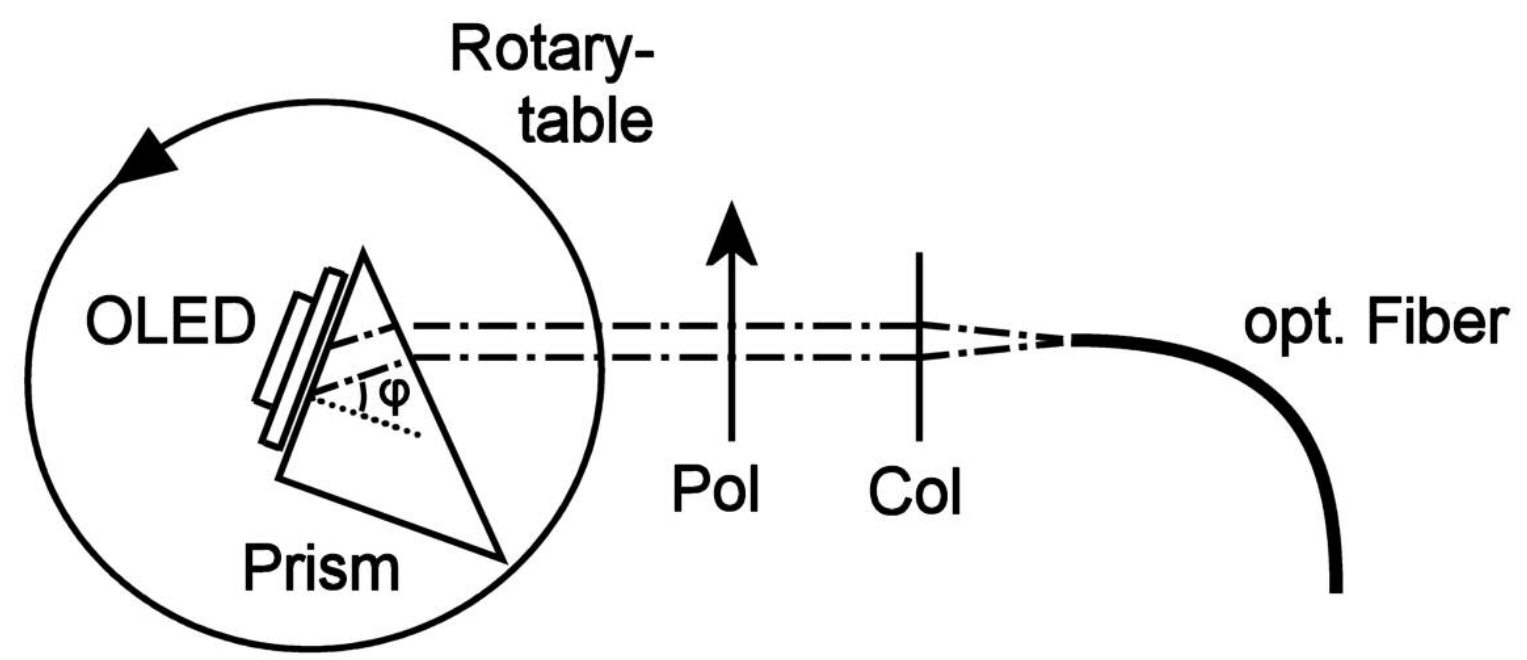

Fig. 2: Measurement setup to record angular dependent emission spectra of OLEDs: a) the device is mounted on a rotary table and light emission through the glass substrate is investigated with a collimator and a polarizing filter. b) The device is mounted onto the cathetus side of a right angle prism. In this configuration, the light leaving through the hypotenuse side is detected. 


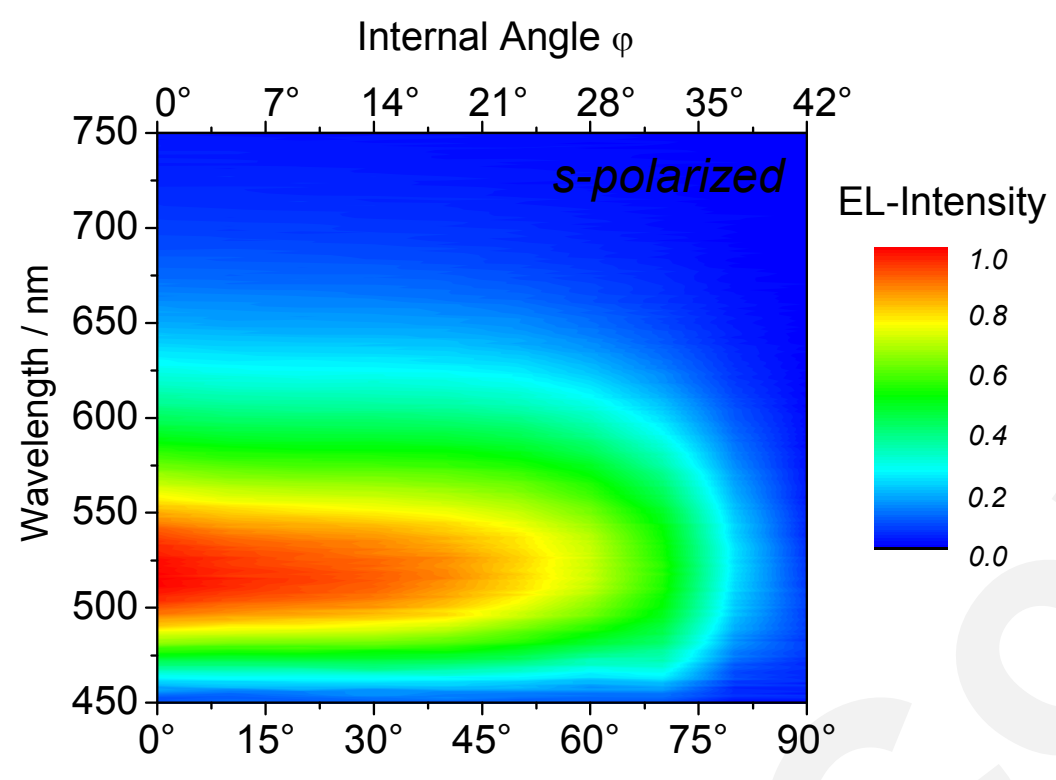

Angle of Emission $\omega$

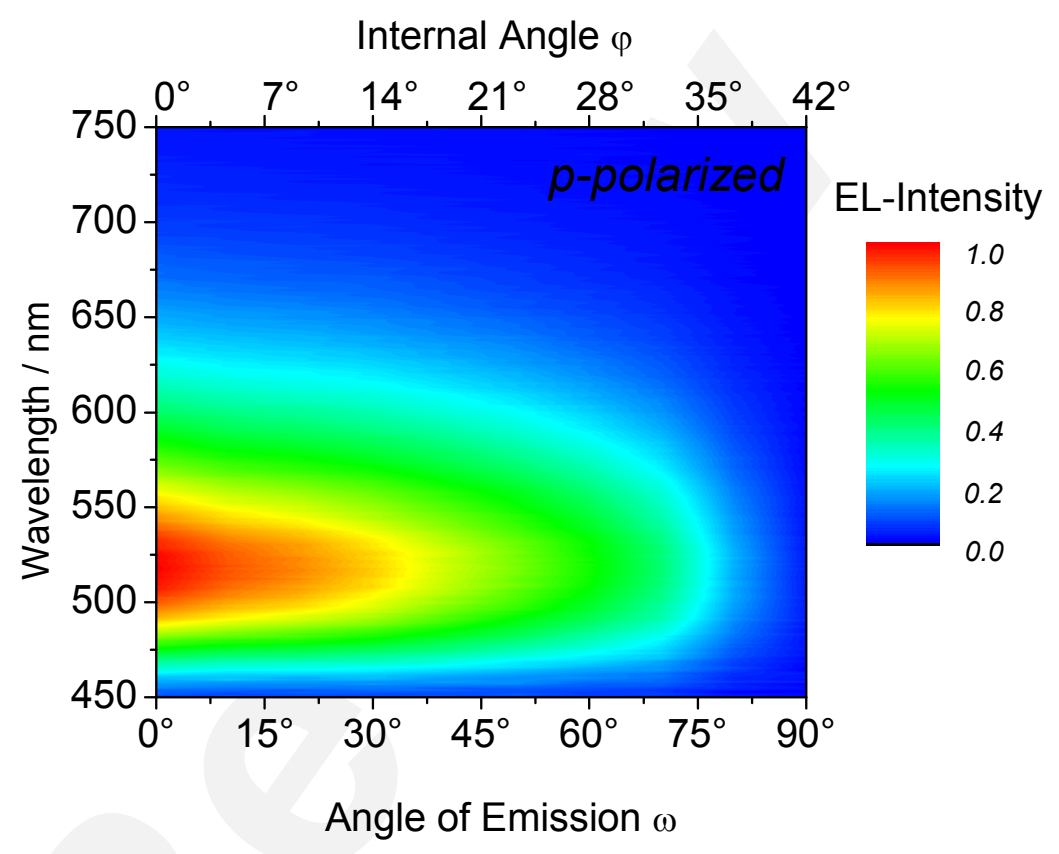

Fig. 3: Angular resolved spectral emission of the reference ITO device (A), measured without prism (top: s-polarized, bottom: p-polarized). 

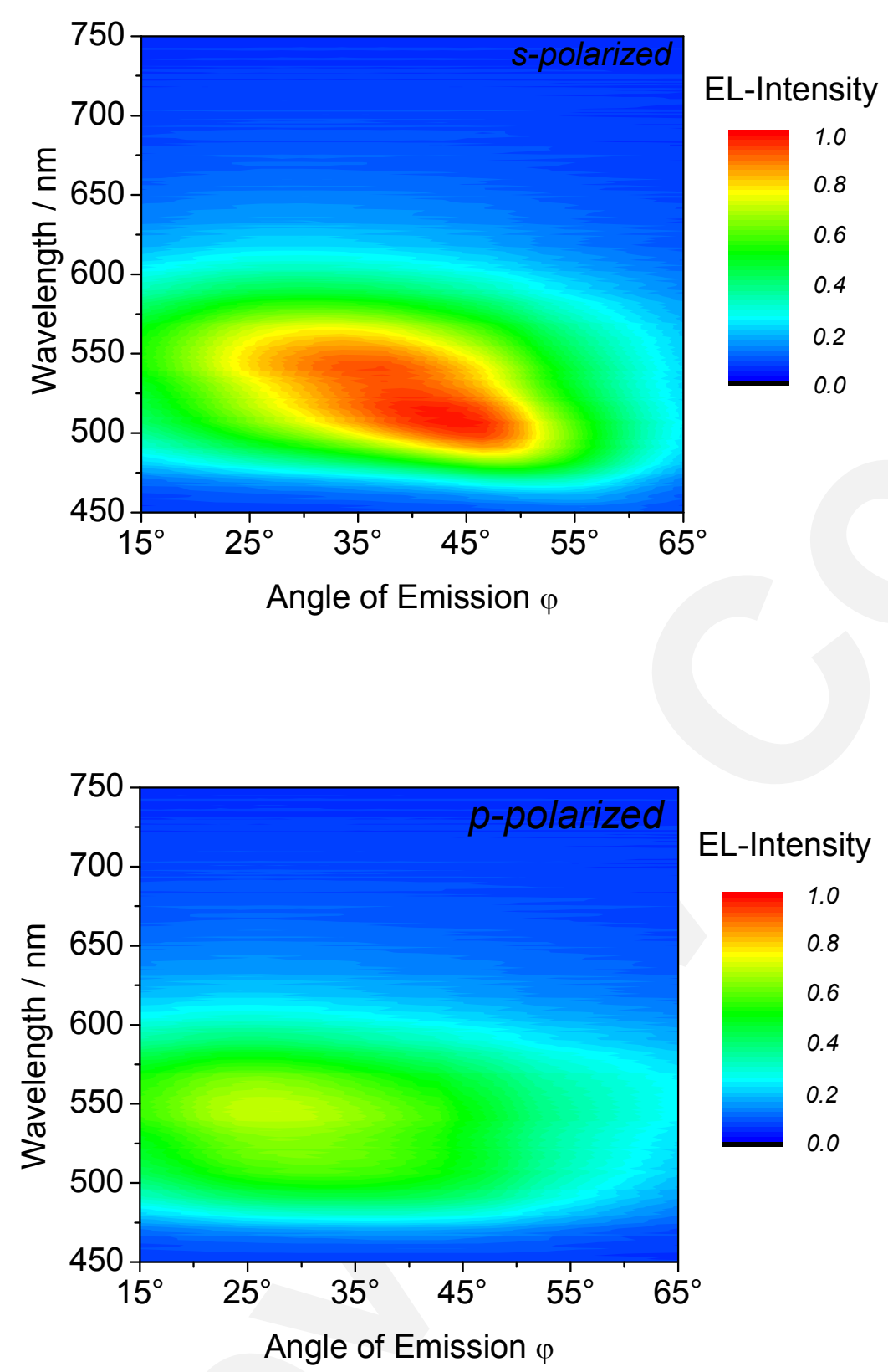

Fig. 4: Angular resolved spectral emission of the reference ITO device (A), measured with the prism (top: s-polarized, bottom: p-polarized). 

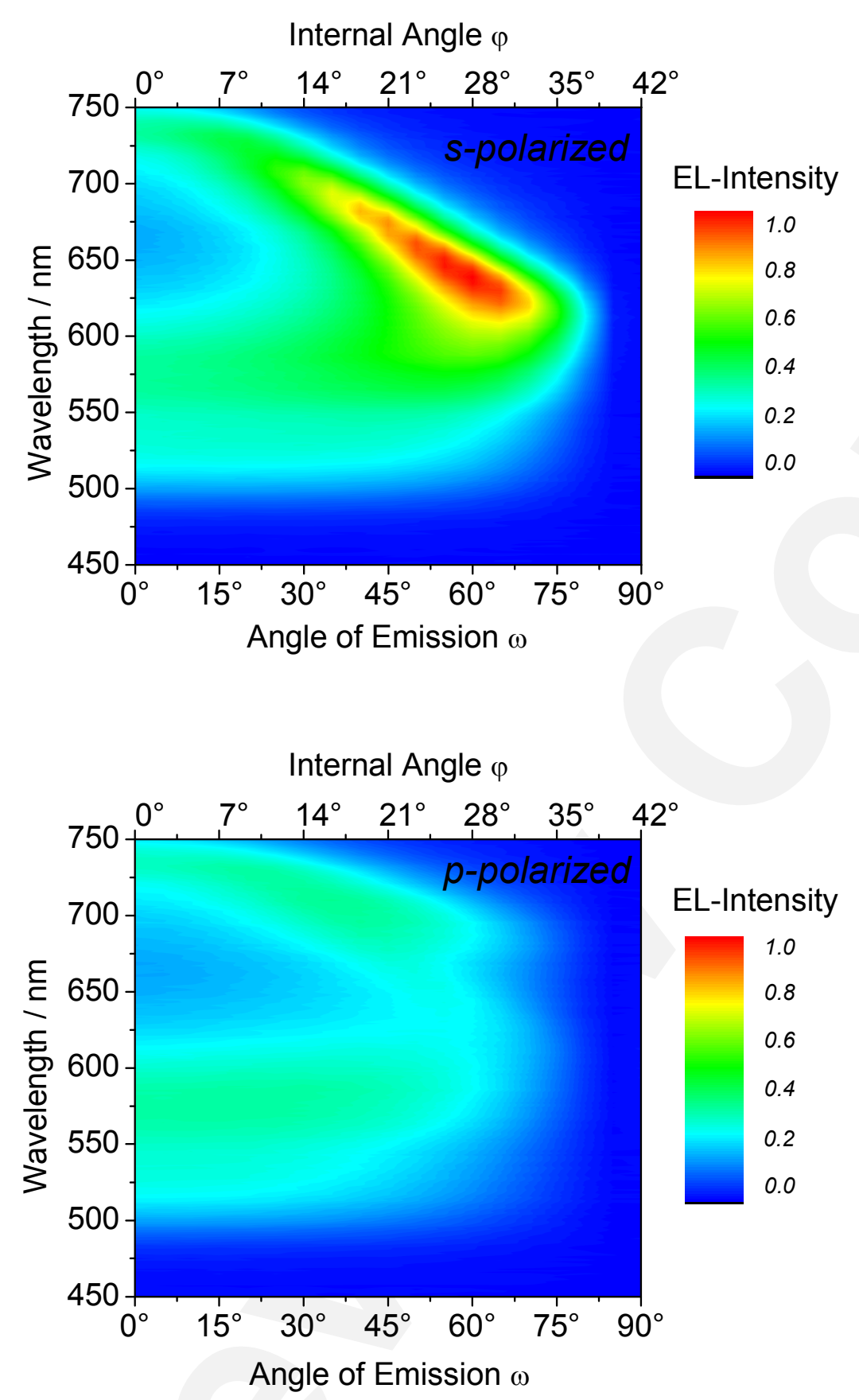

Fig. 5: Angular resolved spectral emission of the leaky-mode device (B), measured without prism (top: s-polarized, bottom: p-polarized). 



Fig. 6: Angular resolved spectral emission of the leaky-mode device (B), measured with the prism (top: s-polarized, bottom: p-polarized). 


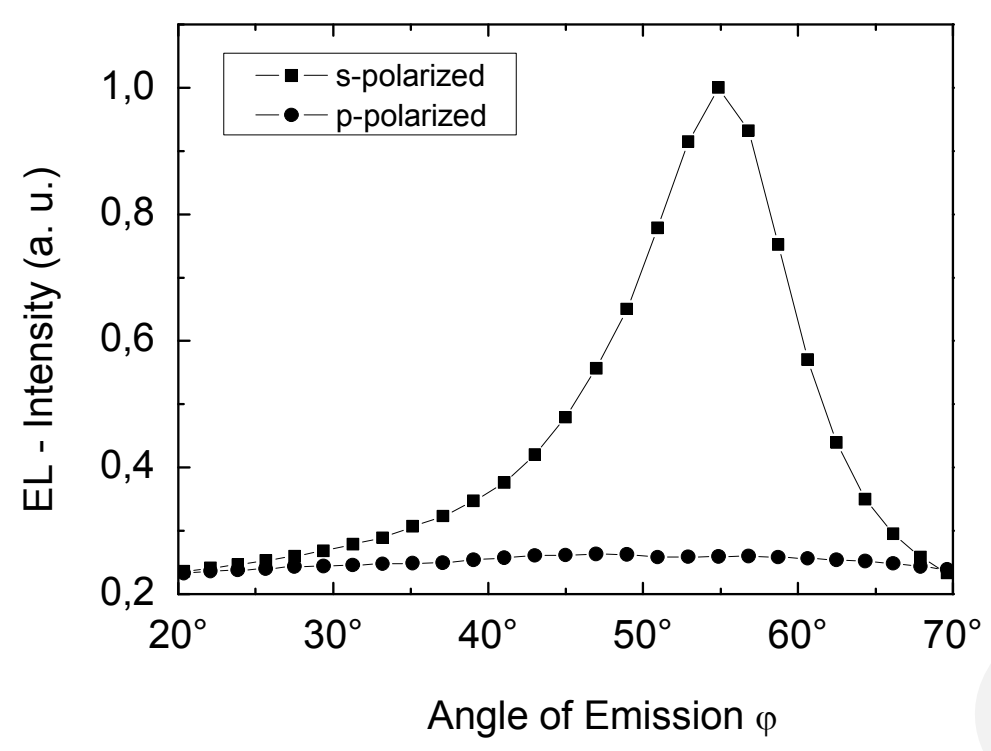

Fig. 7: Angle dependent electroluminescence intensity of the leaky-mode device (B) at an emission wavelength of $550 \mathrm{~nm}$.

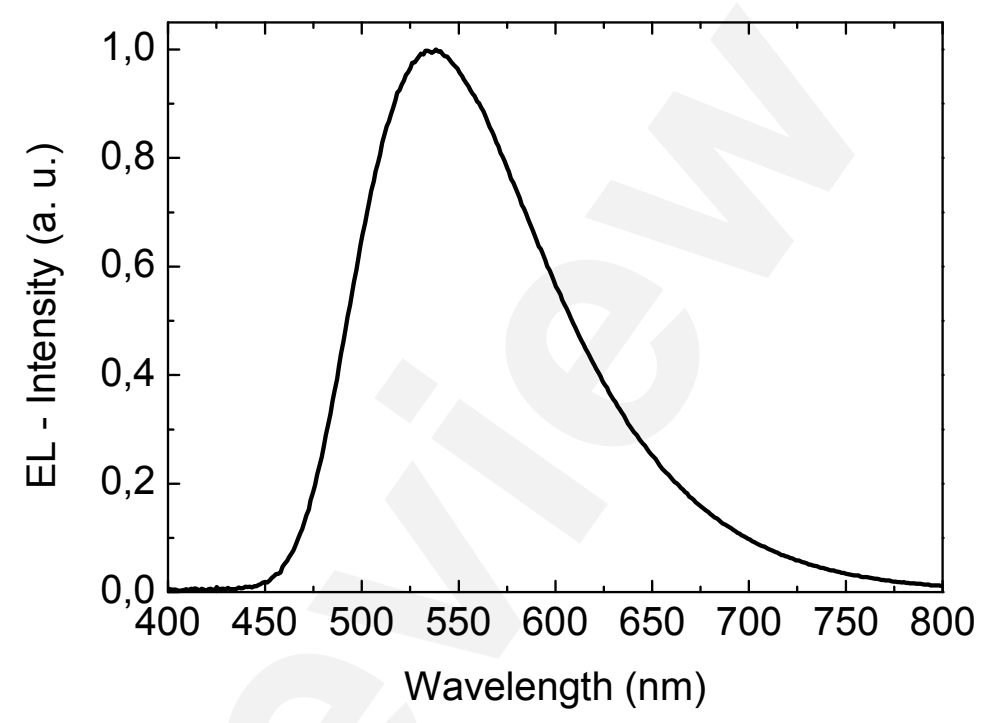

Fig. 8: Emission spectrum of the reference ITO device (A) in forward direction. 

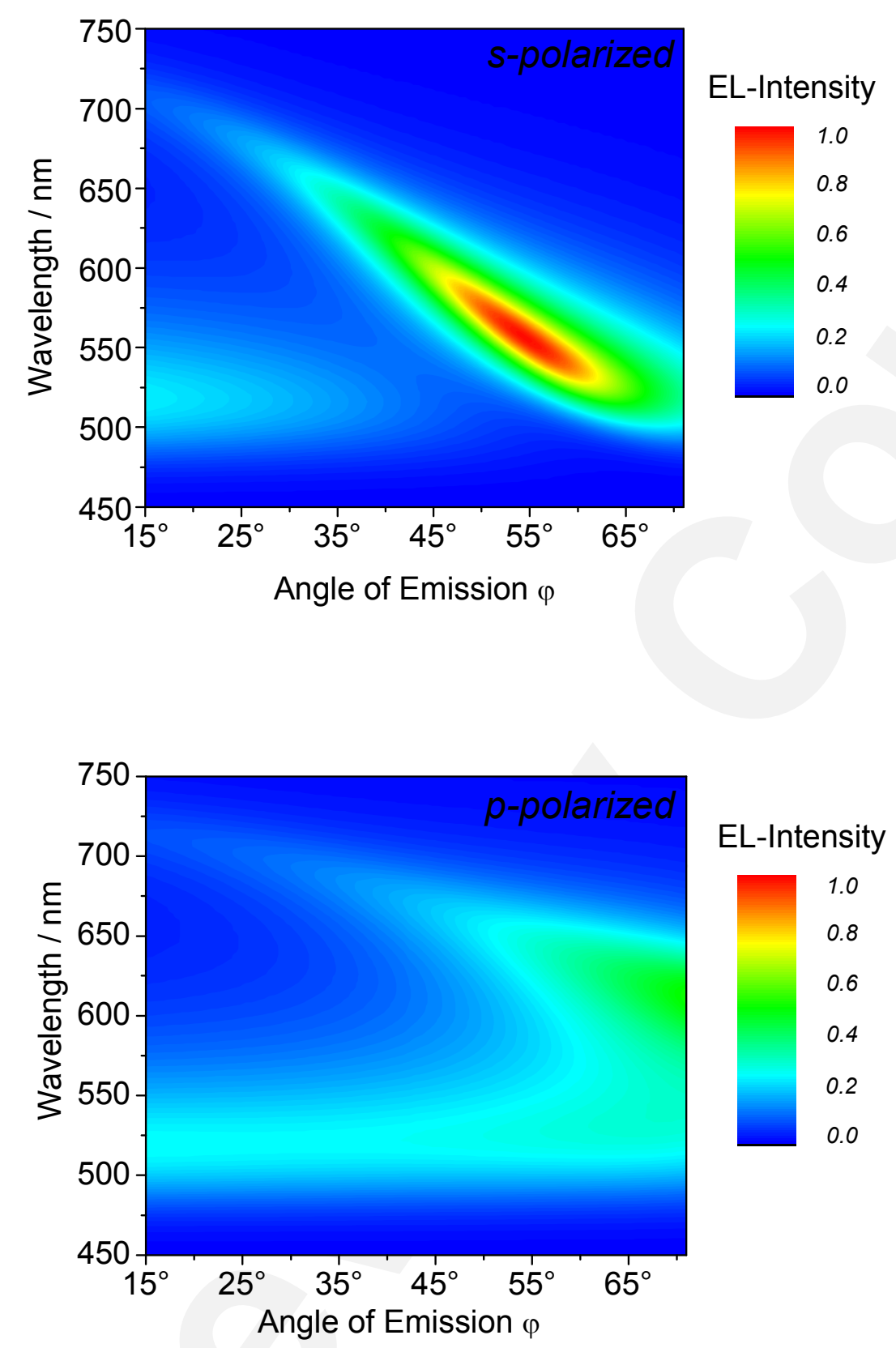

Fig. 9: Simulated angle dependent light emission of the leaky-mode device (B) (top: spolarized, bottom: p-polarized). 